\title{
Re: The Combination of Penile Revascularization Surgery with Penile Corrective Techniques as an Alternative to Prosthesis Implantation in Patients with Peyronie's Disease Accompanied by Erectile Dysfunction: Long-Term Results
}

\author{
Kayigil 01, Okulu E², Akdemir F³, Cakici OU² \\ 'Yildirim Beyazit University Faculty of Medicine, The Urology Clinics of Ankara Ataturk Training and Research Hospital, Ankara, Turkiye \\ ${ }^{2}$ The Urology Clinics of Ankara Ataturk Training and Research Hospital, Ankara, Turkiye \\ ${ }^{3}$ The Urology Clinics of Terme State Hospital, Samsun, Turkiye
}

Int J Impot Res 2017. doi: 10.1038/s41443-017-0012-4.

\section{EDITORIAL COMMENT}

In this study, Kayıgil et al. reported a new surgical approach for the treatment of Peyronie's disease that combines penile revascularization with penile corrective techniques which is an effective alternative to penile prosthesis implantation. In a total of 60 patients, saphenous vein grafts were used in 55, penile dorsal grafts in five, multiple plication and imbrication sutures in 20 , urethra dissection in five, and penile disassembly in one patient. None of the patient had perioperative or postoperative complications and complete penile straightening was achieved in all patients. The mean follow-up period was 18 months. Most patients (53/60) expressed satisfaction with the outcomes of the surgery and reported improvements in their quality of life. According to the data, the authors report excellent outcomes. However, only patients fulfilling very strict inclusion criteria were enrolled and $80 \%$ of them had no risk factors such as atherosclerotic disease, smoking, and advanced age. This technique could be an alternative to penile implant surgery in selected patients.

Emre Bakırcıoğlu, MD

${ }^{\circ}$ Copyright 2018 by the Association of Urological Surgery / Journal of Urological Surgery published by Galenos Publishing House. 